\title{
Mass Spectrometry Based Cancer Classification Using Fuzzy Fractal Dimensions
}

\author{
Tuan D. Pham \\ Bioinformatics Applications Research Center \\ School of Mathematics, Physics, and Information Technology \\ James Cook University \\ Townsville, QLD 4811, Australia \\ tuan.pham@jcu.edu.au
}

\begin{abstract}
Cancer classification using high-throughput mass spectrometry data for early disease detection and prevention has recently become an attractive topic of research in bioinformatics. Recently, several studies have shown that the synergy of proteomic technology and pattern classification techniques is promising for the predictive diagnoses of several cancer diseases. However, the extraction of some effective features that can represent the identities of different classes plays a critical factor for any classification problems involving the analysis of complex data. In this paper we present the concept of a fuzzy fractal dimension that can be utilized as a novel feature of mass spectrometry (MS) data. We then apply vector quantization (VQ) to model the class prototyes using the fuzzy fractal dimensions for classification. The proposed methodology was tested with an MS-based ovarian cancer dataset. Using a simple VQ-based classification rule, the overall average classification rates of the proposed approach were found to be superior to some other methods.
\end{abstract}

Keywords: Feature extraction, fuzzy fractal dimension, fuzzy c-means, vector quantization, mass spectrometry data, cancer classification.

\section{Introduction}

Proteomics has increasingly been a major role in the discovery of disease pathways and biomarkers for new drug treatment and development [12. In comparison with transcriptional profiling in functional genomics, proteomics has some obvious advantages in that it provides a more direct approach to studying cellular functions because most gene functions are characterized by proteins, and more insight on exploring gene functions by studying protein expression.

The identities of expressed proteins in a protemome can be determined by protein separation, identification, and quantification. Protein separation methods involve two-dimensional gel electrophoresis followed by gel image processing. Once proteins are separated, protein differential expression can be characterized using mass spectrometry (MS) which is a high-resolution technique for determining molecular masses. A mass spectrometry dataset consists of relative intensities at chromatographic retention time and the ratios of molecular mass over charge

L. Rueda, D. Mery, and J. Kittler (Eds.): CIARP 2007, LNCS 4756, pp. 614 623, 2007.

(C) Springer-Verlag Berlin Heidelberg 2007 
$(\mathrm{m} / \mathrm{z})$. Thus the mass spectrum for a sample is a function of the molecules and used to test for presence or absence of one or more molecules which may relate to a diseased state or a cell type. Proteomic patterns have recently been used for early detection of cancer progressions [34]. Obviously, early detection of such diseases has the potential to reduce mortality. In fact, it has been forseen that advances in mass-spectrometry based diagnostics may lead to a new revolution in the field of molecular medicine [5]4]6].

Several methods for classification of normal and diseased states using mass spectrometry data have been recently developed. Petricoin et al. 3. applied cluster analysis and genetic algorithms to detect early stage ovarian cancer using proteomic spectra in serum generated by mass spectrometry to distinguish neoplastic from non-neoplastic disease within the ovary. Lilien et al. 7] applied principal component analysis and a linear discriminant function to classify ovarian and prostate cancers using whole spectrum surface-enhanced laser desorption/ioinization time of flight (SELDI-TOF) mass spectronometry data of human serum. Wu et al. 8 compared the performance of several methods for the classification of mass spectrometry data. These classification methods include linear discriminant analysis, quadratic discriminant analysis, $k$-nearest neighbor algorithm, bagging and boosting classification trees, support vector machine, and random forest. The authors tested these methods against ovarian cancer and comtrol serum samples obtained from the National Ovarian Cancer Early Detection Program clinic at the Northwestern University hospital (USA) and found that the random-forest based classifier outperformed other methods in the discrimination of normal individuals from cancer patients based on mass spectrometry data.

Tibshirani et al. 9] proposed a probabilistic approach for sample classification from protein mass spectrometry data. These authors applied the so-called peak probability contrast technique to determine a set of common peaks of the ovarian cancer MALDI-TOF (matrix-assisted laser desoprtion and ionization time-of-flight) data. Based on the assumption that mass spectrometry involves complex functional data where the features of interest are the peak signals, Morris et al. [10 applied wavelet transforms and peak detection for feature extraction of MS data. This procedure consists of two steps as follows. Firstly the MS peaks are extracted; and secondly the resulting mass spectral peaks are then quantified. Yu et al. [11] developed a method for dimensionality reduction for high-throughput MS data. This method consists of four steps for data preprocessing based on binning, Kolmogorov-Smirnov test (KS-test), restriction of coefficient variation, and wavelet transforms. These authors applied the support vector machines (SVM) to train the wavelet coefficients of an ovarian high-resolution SELDI-TOF dataset, and reported that the SVM based classifier outperformed several other classification algorithms such as voted perceptron, discriminant analysis, decision tree analysis, naive Bayes, bagging and boosting classification trees, and random forest.

In this paper, we introduce the concept of a fuzzy fractal dimension and its vector quantization for extracting a novel feature of mass spectrometry signals 
and classification respectively. Application of such computational framework has never been explored before for the analysis of proteomic data.

\section{Feature Extraction by Fuzzy Fractal Analysis}

The fractal dimension 12 is a mathematical expression of the space filling properties of an object whose concept leads to many different types of fractal dimensions [13]. The simplest form of fractal dimensions is the self-similarity dimension. The self-similarities of the line, square, and cube are equal to 1,2 , and 3 respectively. Consider a geometrically self-similar fractal object which consists of line segments. If each line segment is divided into $M$ smaller line segments, then $N$ smaller objects are produced. Furthermore, if the object is geometrically self-similar, then each of the objects of smaller sizes is an exact but reduced size copy of the whole object. The self-similarity dimension $d$ is then expressed as [13]

$$
N=M^{d}
$$

which can be written in a another form as

$$
d=\frac{\log (N)}{\log (M)}
$$

Because the self-similarity dimension requires that each smaller subject formed by the division of the whole object must be an exact copy of the whole object, it can only be used to study objects that are geometrically self-similar. Such a fractal dimension is not very useful for analyzing many real objects that usually have irregular shapes. Thus more general fractal dimensions have been developed as more general forms of the fractal dimension. Two such popular forms are known as the capacity and the Hausdorff dimensions. The capacity of an object can be determined by covering it with balls of a radius $r$. The smallest number of balls $N(r)$ that covers all the parts of the object is counted. Then the radius of the previous balls is reduced and again $N(r)$ is counted. The capacity is the value of $\log N(r) / \log (1 / r)$ in the limit as $r$ shrinks to 0 . The relationship of the capacity and the self-similarity dimensions is that if $M=1 / r$, then $N=M^{d}$. The Hausdorff-Besicovitch dimension is similar, but not identical, to the capacity dimension. In the capacity dimension, the object is covered with the number of balls $N(r)$ of a given radius $r$; whereas in the Hausdorff dimension, the object is covered with sets.

There are two general principles of the concept of the fractal dimension: the scaling dimension and the Hausdorff dimension [14. The first principle, which is often called the telescope-microscope principle, states that the decrease of the measuring scale of an object by a factor $s$ is equivalent to the increase of the measuring scale of the object by the factor $s$. The second principle states that any measurement of the "mass" of a fractal of the Hausdoff dimension $d$ contained in a box of side $s$ is proportional to $s^{d}$. There are many natural ways for computing the mass of a fractal set such as the box counting method [13, 
the area-perimeter method [15], and the mass-radius method [12]. We develop herein a new method for obtaining the fractal dimension using the framework of the fuzzy $c$-means algorithm, that can be useful for extracting some novel feature of mass spectrometry data and easily implemented by the vector quantization technique for pattern classification.

Let $M_{f c}$ be the fuzzy $c$-partition space, and $J: M_{f c} \times \mathcal{R}^{c p} \rightarrow \mathcal{R}^{+}$be [17]

$$
J_{m}(\mathbf{U}, \mathbf{v})=\sum_{k=1}^{n} \sum_{i=1}^{c}\left(u_{i k}\right)^{m}\left(d_{i k}\right)^{2}
$$

where $\mathbf{U} \in M_{f c}$ is a fuzzy partition of $\mathbf{X}=\left(\mathbf{x}_{1}, \mathbf{x}_{1}, \ldots, \mathbf{x}_{n}\right) ; \mathbf{v}=\left(\mathbf{v}_{1}, \mathbf{v}_{2}, \ldots, \mathbf{v}_{c}\right)$ $\in \mathcal{R}^{c p}$ with $\mathbf{v}_{i} \in \mathcal{R}^{p}$ is the cluster center of $u_{i}, 1 \leq i \leq c ; d_{i k}=\left\|\mathbf{x}_{k}-\mathbf{v}_{i}\right\|$ and $\|\cdot\|$ is any inner product induced norm on $\mathcal{R}^{p}$; and $m \in[1, \infty)$.

Observation of (3) reveals that $d_{i k}$ is the the measure of dissimilarity between each data point $\mathbf{x}_{k}$ and fuzzy cluster center $\mathbf{v}_{i}$, whose squared distance is then weighted by the term $\left(u_{i k}\right)^{m}$. Thus, $J_{m}$ is a square error clustering function, and solutions for this fuzzy clustering algorithm is to

$$
\operatorname{minimize}_{M_{f c} \times \mathcal{R}^{c p}} J_{m}(\mathbf{U}, \mathbf{v})
$$

There exists an infinite family of fuzzy clustering algorithms - one for each $m \in[1, \infty)$ - via the conditions for solutions of (4).

In the fuzzy $c$-means (FCM) algorithm; $c$, the number of clusters, needs to be given. In many practical cases, $c$ is unknown. It is reasonable to expect cluster substructure at more than one value of $c$, and therefore necessary to estimate the most plausible value of $c$ for the cluster analysis. This problem is known as cluster validity. It is very difficult to formulate the cluster validity problem in a mathematically tractable manner, because the basic question is imposed on the definition of a cluster. For fuzzy clustering, one should examine which pairs of fuzzy groups/classes overlap, and this leads to the question of how fuzzy a fuzzy $c$-partition is. A heuristic solution to this problem is to calculate the measure of fuzziness in $\mathbf{U}$, and then assign $c$ as the most valid value that has the least fuzzy partitions.

The first functional designed for cluster validity measure is the partition coefficient [17. This partition coefficient of a fuzzy $c$-partition of $\mathbf{U} \in M_{f c}$ of $\mathbf{X}$ is expressed as

$$
F(c)=\frac{1}{n} \sum_{k=1}^{n} \sum_{i=1}^{c}\left(u_{i k}\right)^{2}
$$

Another equivalent expression for (5) that emphasizes various properties of $F$ is the Euclidean inner product for two matrices $\mathbf{I}, \mathbf{J} \in \mathbf{V}_{c n}$ is $\langle\mathbf{I}, \mathbf{J}\rangle=\operatorname{Tr}\left(\mathbf{I} \mathbf{J}^{T}\right)$, where $T r$ is the trace of a matrix, and $\mathbf{J}^{T}$ is the transpose of $\mathbf{J}$. And (5) has alternative forms

$$
F(c)=\frac{\operatorname{Tr}\left(\mathbf{U} \mathbf{U}^{T}\right)}{n}=\frac{<\mathbf{U}, \mathbf{U}>}{n}=\frac{\|\mathbf{U}\|^{2}}{n}
$$


Now it can be analyzed that: if $F(c)=1$ then $\mathbf{U}$ contains no fuzzy clusters ( $\mathbf{U}$ consists of only zeros and ones); if $F(c)=1 / c$ (all elements in $\mathbf{U}$ is equal to $1 / c$ ) then $\mathbf{U}$ is completely fuzzy; and in general $1 / c \leq F(c) \leq 1$. As $F(c)$ increases, the partition of the data sets is more effective. Thus the formal strategy for selecting the most valid $c^{*}$ is as follows. Let $\Omega_{c}$ represents any finite set of optimal U's $\in M_{f c}$, and $c=2,3, \ldots, n-1$. The optimal $c^{*}$ is determined by direct search

$$
c^{*}=\arg \max _{c}\left[\max _{\Omega_{c}} F(c)\right]
$$

An observation of the relationship between $c$ and $F(c)$ reveals that $F(c)$ can be interpreted as a type of the measure of the fuzziness or the "fuzzy mass" of the partition space $\mathbf{U}$ as a function of the number of clusters of size $c$. Based on the second principle of dimension for fractals, $F(c)$ is equivalent to the approximate power law

$$
F(c) \propto c^{d_{f c m}}
$$

where $d_{f c m}$ is called the FCM-based fractal dimension or the fuzzy fractal dimension (FFD) of $\mathbf{U}$, which can be determined as

$$
d_{f c m}=\lim _{c \rightarrow c^{*}} \frac{\log F(c)}{\log c}
$$

It can be further shown that if the plot of $\log F(c)$, the vertical axis, versus $\log c$, the horizontal axis, is represented by a straight line using the method of least squares; then a straight line in a $x-y$ diagram can be expressed as

$$
\log F(c)=d_{f c m} \log c+b
$$

where $d_{f c m}$ is the slope of the line, and $b$ is the intercept of the fitting line with the vertical axis. Alternatively,

$$
F(c)=b c^{d_{f c m}}
$$

Thus, the fuzzy fractal dimension $d_{f c m}$ expression in (9), in the limit where $c$ approaches $c^{*}$, can be determined as the slope of the plot of $\log F(c)$ versus $\log c$. The basic idea is that if an object is self-similar, then the slope of $\log F(c)$ versus $\log c$ is the same as the $\operatorname{limit}$ of $\log F(c) / \log c$ as $c$ approaches $c^{*}$. However, the determination of the slope is much easier than $c^{*}$.

For recognition or classification of complex patterns, it has been reported that the use of a single fractal dimension is not sufficient enough to obtain good results; and therfeore suggested multifractal models [16]. Based on the minimization of $J_{m}$ expressed in (4), an infinite set of the FCM-based fractal dimensions $\mathcal{D}_{f c m}$ can be obtained in terms of the weighting exponent $m$ :

$$
\mathcal{D}_{f c m}=\bigcup_{m=1}^{\infty} d_{f c m}^{m}
$$

To obtain a finite vector of fuzzy fractal dimensions, we set the range for $m$ with some discrete values being greater than one (as $m=1$, the FCM algorithm becomes a hard-clustering case). We now turn our discussion on the 
implemenation of the fuzzy fractal dimensions for pattern classification using vector quantization approach in the next section.

\section{Classification Using FFD-Based Vector Quantization}

To obtain the set of codewords or codevectors which can be modeled as the prototype of a certain class, we apply the method for quantizing the vectors of the fuzzy fractal dimensions. Let $\mathbf{a}_{t}$ be a vector of fuzzy fractal dimensions; and the codebook of the vectors of fuzzy fractal dimensions be $C=\left\{\mathbf{c}_{1}, \mathbf{c}_{2}, \ldots, \mathbf{c}_{L}\right\}$, where $\mathbf{c}_{j}=\left(c_{j 1}, c_{j 2}, \ldots, c_{j p}\right), j=1,2, \ldots, L$ are codewords, Each codeword $\mathbf{c}_{j}$ is assigned to an encoding region $R_{j}$ in the partition $\left\{R_{1}, R_{2}, \ldots, R_{L}\right\}$. The source vector $\mathbf{a}_{t}$ can be represented by the encoding region $R_{n}$ and expressed by

$$
V\left(\mathbf{a}_{t}\right)=\mathbf{c}_{n}, \text { if } \mathbf{a}_{t} \in R_{n}
$$

The main idea of the vector quantization (VQ) is to find an optimal codebook such that for a given training set and a codebook size, the average distortion in representing each vector $\mathbf{a}_{t}$ by the closest codeword $\mathbf{c}_{n}$ is minimum. One of the most popular methods for VQ design is the LBG (Linde, Buzo and Gray) algorithm 18. The LGB-VQ method requires an initial codebook, and iteratively bi-partitions the codevectors based on the optimality criteria of nearest-neighbor and centroid conditions until the number of codevectors is reached.

For the particular study, the MS cancer classification system based on the fuzzy fractal analysis and VQ-codebook approach works as follows. In the training phase, the MS signals are analyzed by the fuzzy fractal analysis to obtain the vectors of fuzzy fractal dimensions (FFD). The training FFD vectors are then quantized using the number of codebooks according to the number of different classes. In the testing phase, an input unknown MS signal, denoted as $s$, is analyzed by the fuzzy fractal analysis resulting in the FFD vector. The dissimilarity between the FFD vector of the unknown sample and each trained codebook is computed. The dissimilarity measure of the unknown sample $s$ and a particular known class represented by the codebook $C^{i}$ is determined using the minimum rule:

$$
D\left(s, C^{i}\right)=\min _{1 \leq j \leq L} D\left(\mathbf{x}_{m}, \mathbf{c}_{j}^{i}\right)
$$

where $D$ is a measure of dissimilarity taken as the $L_{2}$ norm, $\mathbf{x}_{m}$ is the FFD vector of the unknown sample $s, \mathbf{c}_{j}^{i}$ is the $j$ FFD-VQ codevector of a particular known class represented by codebook $C^{i}$.

The unknown sample $s$ is assigned to class $i^{*}$ if the dissimilarity measure of its FFD vector $\mathbf{x}_{m}$ and the FFD-VQ codebook $\mathbf{C}^{i}$ is minimum, that is

$$
\begin{aligned}
& \operatorname{assign} s \text { to class } i^{*} \text { if } \\
& i^{*}=\arg \min _{i} D\left(s, C^{i}\right)
\end{aligned}
$$




\section{Experiment}

The ovarian high-resolution SELDI-TOF mass spectrometry dataset, which can be obtained from the FDA-NCI Clinical Proteomics Program Databank was used to test the proposed approach. The dataset was generated using a nonrandomized study set of ovarian cancers and control specimens on an ABI Qstar fitted with a SELDI-TOF source to study ovarian cancer case versus high-risk control. The dataset consists of 100 control samples and 170 cancer samples. Figures 1 and 2 show the typical MS signals of ovarian control and ovarian cancer respectively.

A vector of 8 fuzzy fractal dimensions is extracted for each sample by using 8 arbitrary values for the FCM weighting exponent $m$ ranging from 1.5 to 5.0 with the interval of 0.5 . Two codebooks of 8 and 16 codevectors were used to generate the two prototypes for the control and cancer classes respectively. The validation of the classification of the proposed approach was designed with similar strategies to those carried out in [11, who applied support vector machine (SVM) for the classification, so that comparisons can be made. The first measure of performance is the $k$-fold cross validation where $k=2,3, \ldots, 10$, and each $k$ fold validation was carried out 1000 times. The second measure of performance is the leave-one-out cross validation.

It is noted that the raw ovarian high-resolution SELDI-TOF dataset used by Yu et al. [1] consists of 95 control samples and 121 cancer samples; while the raw ovarian high-resolution SELDI-TOF dataset we used to test the performance of the proposed approach has 100 control samples and 170 cancer samples. In the leave-one-out cross validation, the proposed method only misclassified 2 control and 2 cancer samples in comparison with 6 control and 2 cancer samples being misclassified by the SVM [11; and 4 control and 2 cancer samples misclassified by the linear predictive coding (LPC)-VQ method 19. It was also reported that the principal component analysis (PCA) was applied to reduce the large number of

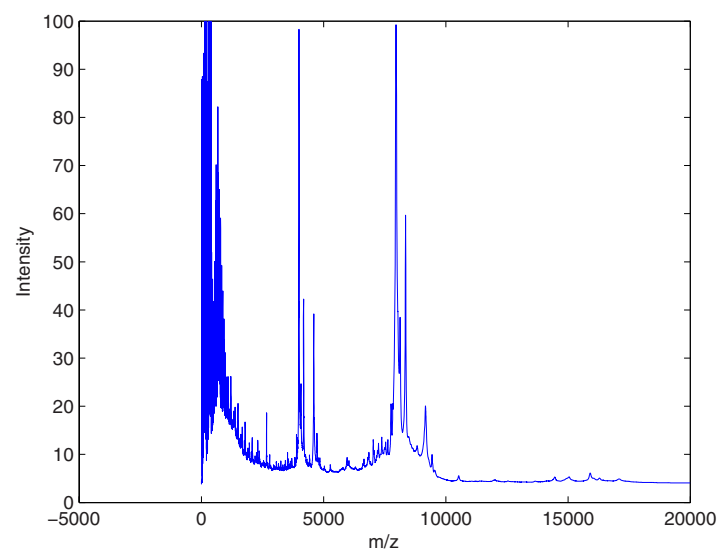

Fig. 1. MS-based ovarian control data 


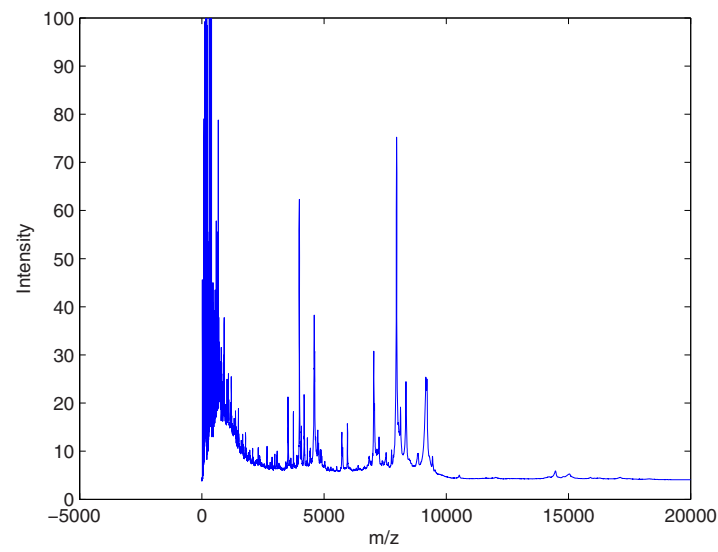

Fig. 2. MS-based ovarian cancer data

Table 1. $k$-fold cross validation results for ovarian cancer data

\begin{tabular}{|c|c|c|c|c|c|c|}
\hline \multicolumn{3}{|c|}{ SVM } & \multicolumn{2}{|c|}{ LPC-VQ } & \multicolumn{2}{|c|}{ FFD-VQ } \\
\hline$k$ & Control meal & Cancer mean & Control mea & Sancer meal & a Control me & Cancer mean \\
\hline 2 & 0.8930 & 0.9492 & 0.9224 & 0.9637 & 0.9314 & 0.9707 \\
\hline 4 & 0.9058 & 0.9722 & 0.9327 & 0.9811 & 0.9450 & 0.9766 \\
\hline 6 & 0.9094 & 0.9760 & 0.9348 & 0.9825 & 0.9531 & 0.9818 \\
\hline 8 & 0.9098 & 0.9784 & 0.9362 & 0.9852 & 0.9570 & 0.9862 \\
\hline 10 & 0.9096 & 0.9801 & 0.9377 & 0.9885 & 0.9564 & 0.9890 \\
\hline
\end{tabular}

features and the PCA-reduced first nine components were used to train the SVMbased classifer [1]. For leave-one-out cross-validation, 9 control and 2 cancer samples were misclassified by the SVM using the PCA reduced dataset. Table 1 shows the mean classification values of the $k$-fold cross validation obtained from the SVM classification on the preprocessed data, the LPC-VQ, and the FFDVQ. Except for $k=2$ and 6 , where the cancer means obtained from the LPCVQ method are highest; the overall results show that the proposed method is most favorable for MS-based ovarian cancer classification than the other two approaches. We also report herein that the Hurst components [16] for all the MS samples are close to one showing the persistency in the MS samples and hence confirms the reliability of the fractal dimensions.

\section{Conclusion}

Fuzzy fractal dimensions (FFDs) have been used as an effective features for classification of cancers using MS data. The use of the fuzzy fractal dimensions 
is suitable for this study because it can capture the inherent spatial information of the MS data, and can be applied to other classification methods.

It has been predicted that the advancement of proteomics pattern diagnostics will lead to a revolution in the field of molecular medicine such as the concept of personalized medicine [20]. It is because this technology not only represents a new model for disease detection but it is also clinically feasible [6]. It is still now the clinical impact of proteomic pattern diagnostics is in the very early stage where the results have not been validated in large trials. Furthermore, recent research outcomes have illustrated the role of MS-based proteomics as an indispensible tool for molecular and cellular biology and for the emerging field of systems biology 2 .

Given these promising results, identifying biomarkers using MS data is a challenging task which requires the combination of the contrast fields of knowledge of modern biology and computational methodology. We have presented in this paper a novel application of fuzzy fractal analysis for extracting a novel feature of mass spectrometry data. The simple decision logic of the VQ-based classification rule is effective for the implementation of the fuzzy fractal dimensions of MS-based cancer data.

\section{References}

1. Griffin, T., Goodlett, D., Aebersold, R.: Advances in proteomic analysis by mass spectrometry. Curr. Opin. Biotechnol. 12, 607-612 (2001)

2. Aebersold, R., Mann, M.: Mass spectrometry-based proteomics. Nature 422, 198 207 (2003)

3. Petricoin, E.F., et al.: Use of proteomic patterns in serum to identify ovarian cancer. Lancet 359, 572-577 (2002)

4. Conrads, T.P., Zhou, M., Petricoin III, E.F., Liotta, I.L., Veenstra, T.D.: Cancer diagnosis using proteomic patterns. Expert Rev. Mol. Diagn. 3, 411-420 (2003)

5. Petricoin, E.F., Liotta, L.A.: Mass spectrometry-based diagnostics: The upcoming revolution in disease detection. Clinical Chemistry 49, 533-534 (2003)

6. Wulfkuhle, J.D., Liotta, L.A., Petricoin, E.F.: Proteomic applications for the early detection of cancer. Nature 3, 267-275 (2003)

7. Lilien, R.H., Farid, H., Donald, B.R.: Probabilistic disease classification of expression-dependent proteomic data from mass spectrometry of human serum. J. Computational Biology 10, 925-946 (2003)

8. Wu, B., Abbott, T., Fishman, D., McMurray, W., Mor, G., Stone, K., Ward, D., Williams, K., Zhao, H.: Comparison of statistical methods for classification of ovarian cancer using mass spectrometry data. Bioinformatics 19, 1636-1643 (2003)

9. Tibshirani, R., Hastie, T., Narasimhan, B., Soltys, S., Shi, G., Koong, A.: Sample classification from protein mass spectrometry, by 'peak probability contrasts'. Bioinformatics 20, 3034-3044 (2004)

10. Morris, J.S., Coombes, K.R., Koomen, J., Baggerly, K.A., Kobayashi, R.: Feature extraction and quantification for mass spectrometry in biomedical applications using the mean spectrum. Bioinformatics 21, 1764-1775 (2005)

11. Yu, J.S., Ongarello, S., Fiedler, R., Chen, X.W., Toffolo, G., Cobelli, C., Trajanoski, Z.: Ovarian cancer identification based on dimensionality reduction for high-throughput mass spectrometry data. Bioinformatics 21, 2200-2209 (2005) 
12. Mandelbrot, B.B.: How long is the coast of Britain? Statistical self-similarity and fractional dimension. Science 156, 636-638 (1967)

13. Liebovitch, L.S.: Chaos Simplified for the Life Sciences. Oxford University Press, New York (1998)

14. Hastings, H.M., Sugihara, G.: Fractals A User's Guide for the Natural Sciences. Oxford University Press, New York (1993)

15. Lovejoy, S.: Area-perimeter relation for rain and cloud areas. Science 216, 185 (1982)

16. Sun, W., Xu, G., Gong, P., Liang, S.: Fractal analysis of remotely sensed images: A review of methods and applications. Int. J. Remote Sensing 27, 4963-4990 (2006)

17. Bezdek, J.C.: Pattern Recognition with Fuzzy Objective Function Algorithms. Plenum Press, New York (1981)

18. Linde, Y., Buzo, A., Gray, R.M.: An Algorithm for Vector Quantization. IEEE Trans. Communications 28, 84-95 (1980)

19. Pham, T.D., Chandramohan, V., Zhou, X., Wong, S.T.C.: Robust feature extraction and reduction of mass spectrometry data for cancer classification. In: Proc. IEEE-ICDM Workshop on Data Mining in Bioinformatics, pp. 202-206 (2006)

20. Ginsburg, G.S., McCarthy, J.J.: Personalized medicine: revolutionizing drug discovery and patient care. Trends Biotechnol. 19, 491-496 (2001) 